\title{
GMR
}

\section{Transcriptome survey of phototransduction and clock genes in marine bivalves}

\author{
X.J. Sun ${ }^{1}$, L.Q. Zhou', J.T. Tian'1, Z.H. Liu', B. Wu', Y.H. Dong' ${ }^{2}$, A.G. Yang ${ }^{1}$ \\ and W.M. $\mathrm{Ma}^{2}$ \\ ${ }^{1}$ Yellow Sea Fisheries Research Institute, Chinese Academy of Fishery Sciences, \\ Qingdao, China \\ ${ }^{2}$ College of Biological and Environmental Sciences, Zhejiang Wanli University, \\ Ningbo, China \\ Corresponding authors: Y.H. Dong / A.G. Yang \\ E-mail: dongyinghui118@126.com / yangag@ysfri.ac.cn \\ Genet. Mol. Res. 15 (4): gmr15048726 \\ Received April 25, 2016 \\ Accepted August 8, 2016 \\ Published October 24, 2016 \\ DOI http://dx.doi.org/10.4238/gmr15048726
}

Copyright $(2016$ The Authors. This is an open-access article distributed under the terms of the Creative Commons Attribution ShareAlike (CC BY-SA) 4.0 License.

\begin{abstract}
Marine animals exhibit a variety of biological rhythms, such as solar and lunar-related cycles; however, our current molecular understanding of biological rhythms in marine animals is quite limited. Identifying and understanding the expression patterns of clock genes from available transcriptomes will help elucidate biological rhythms in marine species. Here, we perform a comprehensive survey of phototransduction and circadian genes using the mantle transcriptome of the scallop Patinopecten yessoensis and compare the results with those from three other bivalves. The comparison reveals the presence of transcripts for most of the core members of the phototransduction and circadian networks seen in terrestrial model species in the four marine bivalves. Matches were found for all 37 queried genes, and the expressed transcripts from the deep sequencing data matched 8 key insect and mammalian circadian genes. This demonstrates the high level
\end{abstract}

Genetics and Molecular Research 15 (4): gmr15048726 
of conservation of the timekeeping mechanism from terrestrial species to marine bivalves. The results provide a valuable gene resource for studies of "marine rhythms" and also further our understanding of the diversification and evolution of rhythms in marine species.

Key words: Transcriptome; Circadian clock; Lunar-related cycles; Circadian rhythm

\section{INTRODUCTION}

Marine ecosystems are governed by a multitude of environmental cycles, such as the light-dark cycle and lunar-related cycles, which are linked to the periodic recurrence of the sun or the moon, and are known as "marine rhythms" (Tessmar-Raible et al., 2011). Most terrestrial organisms possess a circadian clock, which controls metabolic reactions and physiological processes according to daily light-dark cycles (Panda et al., 2002; Schrago, 2006; Golombek and Rosenstein, 2010; Shi and Zheng, 2013). In addition to the circadian rhythm, marine organisms also exhibit lunar-related rhythms that are regulated by lunar periodicity (Ikegami et al., 2014). In contrast to the circadian rhythm, lunar cycles in marine organisms are most prominently involved in reproductive events, such as synchronous mass spawning (Tessmar-Raible et al., 2011). Lunar-controlled reproductive rhythms are widespread and are of fundamental importance for many marine organisms, including corals, sea urchins, mollusks, and fishes (Vize, 2009; Fukushiro et al., 2011; Ikegami et al., 2014). For these marine species, the lunar cycles provide a steady timeframe that can be used for developmental synchronization and mass spawning, even across widespread populations (Tessmar-Raible et al., 2011). For example, in order to ensure successful fertilization, spawning corals utilize an extraordinarily accurate timing system to reproduce according to solar and lunar cycles (Vize et al., 2005). As reported, many species spawn on just one or two evenings per year in a time window only 20-30 min long, which is thought to be controlled by both lunar and solar cycles (Levitan et al., 2004; Vize, 2009).

A number of molecular components involved in circadian rhythms have now been discovered, including the circadian locomotor output cycles kaput (clock), period (per), timeless (tim), and cryptochrome (cry; Wager-Smith and Kay, 2000; Golombek and Rosenstein, 2010; Pairett and Serb, 2013). However, the molecular understanding of circadian rhythms is almost exclusively related to terrestrial species. A number of studies have revealed that the expression of some clock genes, such as per, cry, and melatonin, is affected by both daylight and moonlight, suggesting interconnections between solar and lunar rhythms in animals (Levy et al., 2007; Sugama et al., 2008; Li et al., 2015a). For marine species, molecular components of "marine rhythms" are essential to understand how marine species detect solar and lunar cycles and translate this into the sequence of physiological processes that result in the maturation of gametes and determine the timing of spawning.

Identification and understanding of the expression patterns of clock-controlled genes (CCGs) will help elucidate "marine rhythms" found in marine ecosystems, improve aquaculture, and enhance conservation of natural populations. As a high-throughput method for transcriptome sequencing, RNA-Seq has been widely applied to uncover genes central to the circadian clock at the transcriptome level (Vize, 2009; Pairett and Serb, 2013; Shoguchi et al., 2013). To explore the potential genes underlying biological rhythms in marine species and investigate how these genes are correlated with terrestrial models, we made use of the

Genetics and Molecular Research 15 (4): gmr15048726 
recent generation of a large expressed sequence tag (EST) dataset developed from the mantle transcriptomes of marine bivalves (Pairett and Serb, 2013; Sun et al., 2015). The analysis allowed us to identify most of the key circadian genes from insects and mammals in the transcriptomes of marine bivalves, which indicated that many of these genes are very highly conserved in the animal kingdom. The findings provide a valuable gene resource for studies of "marine rhythms" and further our understanding of the diversification and evolution of rhythms in marine species.

\section{MATERIAL AND METHODS}

\section{Data collection}

Mixed samples of mantle tissue from six scallops of Patinopecten yessoensis produced a total of 55.9 million reads using Illumina paired-end sequencing (Sun et al., 2015), which were used in this study. The raw data are available online at the Short Read Archive of the National Center for Biotechnology Information's (NCBI) trace archive (GenBank accession No. SRP046039). The sequencing data were filtered and assembled as described previously (Sun et al., 2015). Gene expression data for mantle tissue of the Pacific oyster, Crassostrea gigas, were available from Zhang et al. (2012) and was downloaded from an online resource (http://www.oysterdb.com/). The sequences of known proteins related to phototransduction and the circadian clock in terrestrial model species were selected and downloaded from the GenBank database, and are listed with their GenBank accession numbers as shown in Table 1.

\section{Identification of phototransduction and circadian genes}

To identify genes related to phototransduction and the circadian clock, two methods were used in the present study: Kyoto Encyclopedia of Genes and Genomes (KEGG) pathway analyses and targeted searches using the NCBI Basic Local Alignment Search Tool (BLAST; http://blast.ncbi.nlm.nih.gov/Blast.cgi). First, the assembled unigene sequences were aligned to the KEGG database using BLASTx (http://www.genome.jp/tools/blast/), with a similarity cut-off of $30 \%$. All unigenes from KEGG pathways of phototransduction and the circadian clock were selected and identified as candidate genes. Second, the unigenes were submitted to BLAST with annotation against NCBI non-redundant protein sequences $(\mathrm{Nr})$ and nonredundant nucleotide sequences $(\mathrm{Nt})$ to arrange transcripts into clusters according to sequence similarities in order to obtain significant annotations (Sun et al., 2015). Identification of phototransduction and circadian genes was further carried out by searching for key words of genes reported in terrestrial species, such as arrestin, calmodulin, guanyl cyclase, inaC, opsin, phosducin, recoverin, clock, period, timeless, and cycle (Table S1). BLAST for known proteins related to phototransduction and the circadian clock in terrestrial species aimed to identify homologs within the mantle transcriptomes of $P$. yessoensis and C. gigas.

Sequence analysis was undertaken using Geneious v.5.5 (http://www.geneious. com), with a cut-off E-value of $10^{-3}$. The unigene with the lowest E-value was selected as the representative when several unique transcripts were assigned to the same reference gene. The identified phototransduction and circadian genes in P. yessoensis were compared to those within the oyster C. gigas and the scallops Placopecten magellanicus and Argopecten irradians, which were previously summarized by Pairett and Serb (2013) from the eye transcriptome.

Genetics and Molecular Research 15 (4): gmr15048726 


\section{RESULTS}

\section{Four light-mediated pathways annotated in the mantle transcriptome}

Of the 259 pathways annotated by KEGG in the mantle transcriptome of $P$. yessoensis, four important pathways related to phototransduction and the circadian clock were found, including phototransduction-fly (KO04745), circadian rhythm (KO04710), circadian entrainment (KO04713), and circadian rhythm-fly (KO04711). Although the pathway of phototransduction (KO04744) previously found in vertebrates was identified by KEGG annotation, it is unlikely to be the key pathway related to phototransduction and the circadian clock in scallops because only two genes (calmodulin and beta-arrestin) were detected to be significantly expressed with a cut-off E-value of $10^{-3}$. In contrast, for the annotated pathway of phototransduction-fly, more than one half of the key genes $(12 / 21)$ were detected to be expressed in the mantle of $P$. yessoensis, such as phospholipase $C$, myosin, protein kinase $C$, troponin C-long, $\mathrm{Ca}^{2+} /$ calmodulin-dependent protein kinase, $\mathrm{G}$ protein, etc. (Table S1). Among the pathways of circadian rhythm, circadian entrainment, and circadian rhythm-fly, there were a total of 67 unique genes identified related to the circadian clock, including phospholipase $C$, adenylate/guanylate cyclase, calmodulin and related proteins, G-protein (alpha, beta, and gamma subunit), adenylate/guanylate kinase, period, clock, cycle, etc. (Table S1).

\section{Target BLAST for genes related to phototransduction and circadian clock}

BLAST for known proteins related to phototransduction and the circadian clock identified 50 homologs, among the 55 known proteins, with E-values less than $10^{-3}$. For the 50 protein genes, different numbers of unigenes were detected as homologs in the mantle transcriptome, ranging from 1 (arrestin) to 280 (inaC; Table 1). Five key phototransduction genes, including $G$ protein, nina $C$, protein kinase $A$, ina $C$, and rhodopsin kinase, were detected to have more than 200 homologous unigenes.

\section{Summary of genes related to phototransduction and the circadian clock in bivalves}

The available expression data made it possible to compare the phototransduction and circadian clock genes among four bivalve species, P. magellanicus, A. irradians, P. yessoensis, and $C$. gigas. The 29 phototransduction and 8 circadian clock genes are summarized for the four bivalve species in Table 2. Most of the known genes were consistently found in $P$. yessoensis and $C$. gigas, having 28/29 phototransduction genes and $8 / 8$ circadian genes expressed in their mantle tissue. Of the phototransduction genes, PDE gamma expression was not identified in $P$. yessoensis, while trp-like expression was not detected in C. gigas (Table 2). For the other two species, 21/29 phototransduction genes and 6/8 circadian genes were found in P. magellanicus, while 9/29 phototransduction genes and 0/8 circadian genes occurred in A. irradians. Based on the transcriptome data, we predicted the model of the circadian clock in scallops. In the first feedback loop, the Clock:Cycle complex activates transcription of repressor genes Per and Tim, and their proteins inhibit the transcriptional activity of Clock:Cycle (Figure 1). In the second feedback loop, because the expression of Rev-erb and Vri are not detected, the Clock:Cycle may activate the transcription of the RAR-related orphan receptor (Ror) and the PAR domain protein $(P d p)$, and their proteins feedback to repress or activate Clock mRNA transcription.

Genetics and Molecular Research 15 (4): gmr15048726 
Table 1. GenBank accession number and BLAST results for known proteins related to phototransduction and the circadian clock in the mantle transcriptome of Patinopecten yessoensis.

\begin{tabular}{|c|c|c|c|c|}
\hline $\begin{array}{l}\text { Pathway } \\
\text { nath }\end{array}$ & Protein name & Species & GenBank accession No. & $\begin{array}{l}\text { BLAST (unigene } \\
\text { numbers) }\end{array}$ \\
\hline \multirow[t]{37}{*}{ Phototransduction } & Arrestin & Drosophila melanogaster & AAF53644 & $\sqrt{ }(1)$ \\
\hline & Arrestin & Homo sapiens & CAA72577 & - \\
\hline & calmodulin & Drosophila melanogaster & NP_725120 & $\sqrt{ }(121)$ \\
\hline & CDSA (CDP diglyceride synthetase) & Drosophila melanogaster & NP_524661 & $\sqrt{ }(1)$ \\
\hline & CNGA (cGMP-gated cation channel alpha) & Homo sapiens & P29973 & $\sqrt{ }(13)$ \\
\hline & CNGA (cGMP-gated cation channel alpha) & Homo sapiens & Q9NQW8 & $\sqrt{ }(8)$ \\
\hline & CNGB (cGMP-gated cation channel beta) & Homo sapiens & Q14028 & $\sqrt{ }(8)$ \\
\hline & RdgA (retinal degeneration A) & Drosophila melanogaster & ADV37647 & $\sqrt{(100)}$ \\
\hline & Rhodopsin, G0-coupled & Mizhuopecten yessoensis & O15974 & $\sqrt{(41)}$ \\
\hline & G-protein, alpha subunit & Homo sapiens & NP_620073 & $\sqrt{ }(12)$ \\
\hline & G-protein, beta subunit & Homo sapiens & AAA35922 & $\sqrt{ }(244)$ \\
\hline & G-protein, beta subunit & Drosophila melanogaster & AAS65363 & $\sqrt{ }(220)$ \\
\hline & G-protein, gamma subunit & Homo sapiens & AAB70039 & $\sqrt{(3)}$ \\
\hline & G-protein, gamma subunit & Drosophila melanogaster & NP_724721 & $\sqrt{ }(2)$ \\
\hline & Opsin & Drosophila melanogaster & AAA28733 & $\sqrt{ }(26)$ \\
\hline & G-protein alpha q subunit & Drosophila melanogaster & NP_725191 & $\sqrt{ }(13)$ \\
\hline & Rhodopsin & Homo sapiens & NP_000530 & $\sqrt{ }(42)$ \\
\hline & G-protein alpha subunit & Homo sapiens & NP_005263 & $\sqrt{ }(10)$ \\
\hline & guanyl cyclase & Homo sapiens & AAA60366 & $\sqrt{(5)}$ \\
\hline & inaD (inactivation no afterpotential D) & Drosophila melanogaster & NP_726260 & $\sqrt{ }(23)$ \\
\hline & ninaC (neither inactivation nor afterpotential C) & Drosophila melanogaster & AAF52504 & $\sqrt{ }(209)$ \\
\hline & $\begin{array}{l}\text { PDE alpha (Rod cGMP-specific 3',5'-cyclic } \\
\text { phosphodiesterase subunit alpha) }\end{array}$ & Homo sapiens & NP_000431 & $\sqrt{ }(19)$ \\
\hline & $\begin{array}{l}\text { PDE beta (Rod cGMP-specific 3',5'-cyclic } \\
\text { phosphodiesterase subunit beta) }\end{array}$ & Homo sapiens & $\mathrm{P} 35913$ & $\sqrt{ }(19)$ \\
\hline & $\begin{array}{l}\text { PDE gamma (Retinal rod rhodopsin-sensitive cGMP 3',5'- } \\
\text { cyclic phosphodiesterase subunit gamma) }\end{array}$ & Homo sapiens & P18545 & - \\
\hline & phosducin & Homo sapiens & CAI13122 & $\sqrt{ }(2)$ \\
\hline & phospholipase C & Drosophila melanogaster & ACZ95198 & $\sqrt{ }(9)$ \\
\hline & PI3K & Drosophila melanogaster & AAN14359 & $\sqrt{ }(9)$ \\
\hline & PI synthase & Drosophila melanogaster & NP_573055 & $\sqrt{ }(1)$ \\
\hline & RdgB (retinal degeneration $\mathrm{B}$ ) & Drosophila melanogaster & ACZ95286 & $\sqrt{(4)}$ \\
\hline & PIP kinase & Drosophila melanogaster & O96838 & $\sqrt{(27)}$ \\
\hline & protein kinase $\mathrm{A}$, gamma subunit & Homo sapiens & AAC41690 & $\sqrt{ }(229)$ \\
\hline & protein kinase $\mathrm{A}$ & Rattus norvegicus & AAQ81631 & $\sqrt{ }(218)$ \\
\hline & inaC (inactivation no afterpotential C) & Drosophila melanogaster & NP_476863 & $\sqrt{ }(280)$ \\
\hline & recoverin & Homo sapiens & NP_002894 & $\sqrt{ }(24)$ \\
\hline & Rhodopsin kinase & Homo sapiens & AAB05929 & $\sqrt{ }(203)$ \\
\hline & TRP (transient receptor potential) & Drosophila melanogaster & AAF56970 & $\sqrt{ }(19)$ \\
\hline & TRPL (trp-like) & Drosophila melanogaster & NP_476895 & $\sqrt{ }(20)$ \\
\hline \multirow[t]{18}{*}{ Circadian clock } & ARNTL & Mus musculus & AAH1 1080.1 & $\sqrt{(11)}$ \\
\hline & casein kinase I epsilon & Gallus gallus & AAP47012.1 & $\sqrt{ }(123)$ \\
\hline & Per & Bulla gouldiana & AF353619 & $\sqrt{ }(3)$ \\
\hline & Clock & Mus musculus & AF146793 & $\sqrt{(12)}$ \\
\hline & clock, isoform A & Drosophila melanogaster & NP_523964.2 & $\sqrt{ }(10)$ \\
\hline & clock, isoform D & Drosophila melanogaster & NP_001014576.1 & $\sqrt{ }(8)$ \\
\hline & clock, isoform $\mathrm{F}$ & Drosophila melanogaster & NP_001014574.1 & $\sqrt{ }(7)$ \\
\hline & Cry & Drosophila melanogaster & AAC 83828.1 & $\sqrt{ }(3)$ \\
\hline & cryl & Mus musculus & AF156986 & $\sqrt{ }(5)$ \\
\hline & cry2 & Mus musculus & AF156987 & $\sqrt{ }(5)$ \\
\hline & Cycle & Drosophila melanogaster & AAF49107.1 & $\sqrt{ }(13)$ \\
\hline & discs overgrown (doubletime) & Drosophila melanogaster & NP_733414.1 & $\sqrt{ }(106)$ \\
\hline & Period & Drosophila melanogaster & BAB15925.1 & - \\
\hline & Period & Crassostrea gigas & EKC24403.1 & $\sqrt{ }(4)$ \\
\hline & period1 & Mus musculus & O35973.2 & $\sqrt{(3)}$ \\
\hline & period, putative & Mytilus galloprovincialis & ABM66066.1 & - \\
\hline & timeless & Mus musculus & BAA76390.2 & $\sqrt{ }(6)$ \\
\hline & timeless & Drosophila melanogaster & CAM59649.1 & - \\
\hline
\end{tabular}

Genetics and Molecular Research 15 (4): gmr15048726 
Table 2. Expression of key phototransduction and circadian clock genes in the mantle or eyes of mollusks.

\begin{tabular}{|c|c|c|c|c|c|}
\hline \multirow[t]{2}{*}{ Pathway } & \multirow{2}{*}{ Gene } & \multicolumn{4}{|c|}{ Mollusk species } \\
\hline & & P. magellanicus & A. irradians & P. yessoensis & C. gigas \\
\hline \multirow[t]{29}{*}{ Phototransduction } & Arrestin & $\sqrt{ }$ & & $\sqrt{ }$ & $\sqrt{ }$ \\
\hline & Calmodulin & $\sqrt{ }$ & $\sqrt{ }$ & $\sqrt{ }$ & $\sqrt{ }$ \\
\hline & CDSA (CDP diglyceride synthetase) & & & $\sqrt{ }$ & $\sqrt{ }$ \\
\hline & CNGA (cGMP-gated cation channel alpha) & $\sqrt{ }$ & & $\sqrt{ }$ & $\sqrt{ }$ \\
\hline & CNGB (cGMP-gated cation channel beta) & $\sqrt{ }$ & & $\sqrt{ }$ & $\sqrt{ }$ \\
\hline & G-protein, alpha subunit & $\sqrt{ }$ & $\sqrt{ }$ & $\sqrt{ }$ & $\sqrt{ }$ \\
\hline & G-protein, beta subunit & $\sqrt{ }$ & $\sqrt{ }$ & $\sqrt{ }$ & $\sqrt{ }$ \\
\hline & G-protein, gamma subunit & $\sqrt{ }$ & & $\sqrt{ }$ & $\sqrt{ }$ \\
\hline & guanyl cyclase & & & $\sqrt{ }$ & $\sqrt{ }$ \\
\hline & inaC (inactivation no afterpotential C) & $\sqrt{ }$ & & $\sqrt{ }$ & $\sqrt{ }$ \\
\hline & inaD (inactivation no afterpotential D) & & & $\sqrt{ }$ & $\sqrt{ }$ \\
\hline & \multicolumn{2}{|l|}{ ninaC (neither inactivationnor afterpotential C) } & $\sqrt{ }$ & $\sqrt{ }$ & $\sqrt{ }$ \\
\hline & Opsin & $\sqrt{ }$ & $\sqrt{ }$ & $\sqrt{ }$ & $\sqrt{ }$ \\
\hline & PDE alpha & $\sqrt{ }$ & & $\sqrt{ }$ & $\sqrt{ }$ \\
\hline & PDE beta & $\sqrt{ }$ & & $\sqrt{ }$ & $\sqrt{ }$ \\
\hline & PDE gamma & & & & $\sqrt{ }$ \\
\hline & Phosducin & 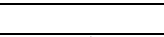 & & $\sqrt{ }$ & $\sqrt{ }$ \\
\hline & phospholipase C & $\sqrt{ }$ & & $\sqrt{ }$ & $\sqrt{ }$ \\
\hline & PI synthase & $\sqrt{ }$ & & $\sqrt{ }$ & $\sqrt{ }$ \\
\hline & PI3K & $\sqrt{ }$ & & $\sqrt{ }$ & $\sqrt{ }$ \\
\hline & PIP kinase & $\sqrt{ }$ & $\sqrt{ }$ & $\sqrt{ }$ & $\sqrt{ }$ \\
\hline & protein kinase $\mathrm{A}$ & $\sqrt{ }$ & $\sqrt{ }$ & $\sqrt{ }$ & $\sqrt{ }$ \\
\hline & RdgA (retinal degeneration $\mathrm{A}$ ) & $\sqrt{ }$ & $\sqrt{ }$ & $\sqrt{ }$ & $\sqrt{ }$ \\
\hline & RdgB & $\sqrt{ }$ & & $\sqrt{ }$ & $\sqrt{ }$ \\
\hline & Recoverin & & $\sqrt{ }$ & $\sqrt{ }$ & $\sqrt{ }$ \\
\hline & Rhodopsin & & & $\sqrt{ }$ & $\sqrt{ }$ \\
\hline & Rhodopsin kinase & $\sqrt{ }$ & & $\sqrt{ }$ & $\sqrt{ }$ \\
\hline & TRP & $\sqrt{ }$ & & $\sqrt{ }$ & $\sqrt{ }$ \\
\hline & TRPL & $\sqrt{ }$ & & $\sqrt{ }$ & \\
\hline \multirow[t]{8}{*}{ Circadian clock } & Arntl & & & $\sqrt{ }$ & $\sqrt{ }$ \\
\hline & casein kinase I & & & $\sqrt{ }$ & $\sqrt{ }$ \\
\hline & Clock & $\sqrt{ }$ & & $\sqrt{ }$ & $\sqrt{ }$ \\
\hline & Cry & $\sqrt{ }$ & & $\sqrt{ }$ & $\sqrt{ }$ \\
\hline & Cycle & $\sqrt{ }$ & & $\sqrt{ }$ & $\sqrt{ }$ \\
\hline & Doubletime & $\sqrt{ }$ & & $\sqrt{ }$ & $\sqrt{ }$ \\
\hline & Period & $\sqrt{ }$ & & $\sqrt{ }$ & $\sqrt{ }$ \\
\hline & Timeless & $\sqrt{ }$ & & $\sqrt{ }$ & $\sqrt{ }$ \\
\hline
\end{tabular}

The expression data for Placopecten magellanicus and Argopecten irradians are summarized from Pairett and Serb (2013). Data for gene expression in the mantle of Crassostrea gigas were downloaded from an online database (http://www.oysterdb.com/). Data for Patinopecten yessoensis are from the current study.

\section{DISCUSSION}

As an important marine species, scallops are present in all of the World's oceans. The mantle of the scallop not only secretes biomineralization proteins along its edge to induce shell formation, but it is also responsible for light-mediated functions and circadian rhythms due to 30-200 single chambered eyes along the mantle edge (Parsons et al., 1992; Pairett and Serb, 2013). In the present study, comparison of the phototransduction and circadian clock genes among four bivalve species indicated that the lack of target genes in scallops of $P$. magellanicus and $A$. irradians may be due to the low read depth in their transcriptomes, which both have less 
than one million reads (114,052 reads for $A$. irradians and 654,002 reads for P. magellanicus) generated from only eye tissue. In contrast, there were a total of 55.9 million reads in our assembled transcriptome of $P$. yessoensis produced from the mantle tissue-containing eyes, in which most phototransduction genes and all key genes related to circadian clock function were detected during this study. Indeed, experimental studies have demonstrated that 10-15 million reads in flies and 20-25 million reads in mice may be sufficient to characterize the majority of the circadian transcripts (Li et al., 2015b). Based on that, $\sim 1 / 10$ of a million reads is insufficient to detect the circadian transcripts in A. irradians in comparison with $\sim 56$ million reads in the mantle transcriptome of $P$. yessoensis.

These results suggest that the number of identified phototransduction and circadian transcripts mainly depends on read depth, rather than tissue type, e.g., eye or mantle. For scallops, although their eyes along their mantle edge are potentially an important entry for light entrainment of the clock, some of the clock genes may originate from extraocular sources in the mantle tissue (Pairett and Serb, 2013). Moreover, scallop eyes are generally so small that they may be insufficient to meet the sample requirement for the next-generation sequencing platform. It is therefore suggested that scallop mantle, including eyes, is the optimal tissue used in the next-generation sequencing platform to identify phototransduction and circadian transcripts, and the requirement of sequencing depth should be at least 10-20 million reads (Li et al., 2015b).

Identification of most phototransduction and circadian clock genes in this study indicates that these genes are highly conserved, not only in insect and mammals, but also in marine bivalves and corals (Shoguchi et al., 2013). Six key genes central to the circadian clock in insect and mammals, including clock, cry, cycle, doubletime (DBT), period (per), and timeless (tim), are consistently detected in three bivalve species, which suggests that the circadian network may also function in marine bivalves. For terrestrial model species, such as mammals and insects, the circadian clock mechanism consists of both negative and positive feedback loops that drive rhythmic expression patterns of core clock components (Lowrey and Takahashi, 2000). In mammals, the negative feedback loop involves a rhythmic transcription of per and cry (Golombek and Rosenstein, 2010). The transcription factors Clock and Bmall interact and activate per and cry expression by binding to an $\mathrm{E}$ box in their promoter regions. PER and CRY proteins form the heterodimeric complex and translocate to the nucleus, which acts on the BMAL1:CLOCK heterodimer to repress its own transcription. The degradation of PER and CRY proteins is phosphorylated by $\mathrm{CK} 1 \varepsilon$ (casein kinase 1 epsilon). For the positive feedback loop, BMAL1:CLOCK activates mRNA expression of nuclear hormone receptor genes Reverb and Ror, and their proteins feedback to negatively and positively regulate Clock and Bmall transcriptions (Golombek and Rosenstein, 2010). Similarly, in the fruit fly, the negative and positive feedback loops also operate in the circadian clock system (Hardin, 2005). The Clock:Cycle heterodimer complex activates transcription of repressor genes Per and Tim (Figure 1). Accumulated PER and TIM proteins form heterodimers to repress the transcriptional activity of the Clock:Cycle. The PER and TIM proteins are phosphorylated by the CK1 $1 \varepsilon$ homolog doubletime, followed by degradation. In another feedback loop, Clock:Cycle activates the transcription of $V r i$ and $P d p$, and their proteins feedback to repress and activate Clock mRNA transcription, respectively (Golombek and Rosenstein, 2010).

Genetics and Molecular Research 15 (4): gmr15048726 


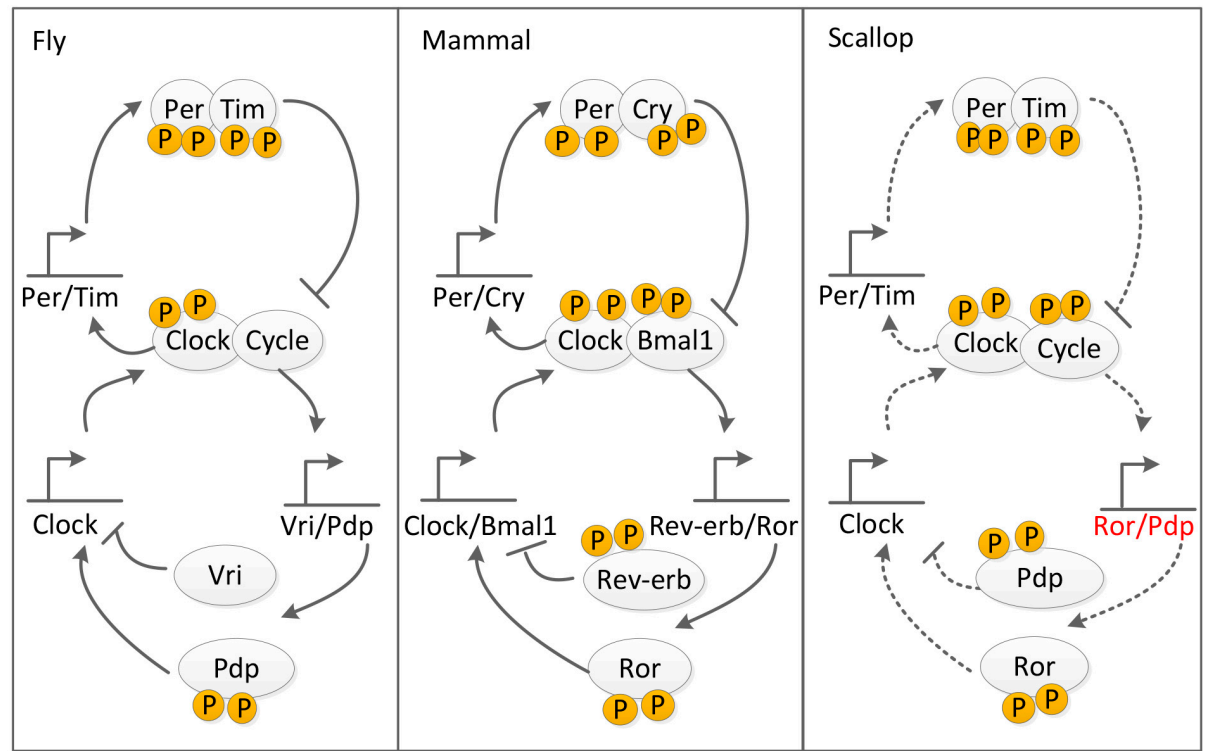

Figure 1. Hypothesized molecular framework of the circadian clock for the Yesso scallop Patinopecten yessoensis compared to that in two model organisms, a fly and a mammal, as summarized by Shi and Zheng (2013). Dashed black lines indicate the hypothetical relationships based on expression data from the current study. P, phosphorylation; Per, Period; Tim, timeless; Cry, Cryptochrome; Pdp, PAR domain protein; Bmal1, Brain and muscle arnt-like protein 1; Vri, Vrille; Ror, receptor-related orphan receptors.

The present results support the inference that the basic framework of clock regulation is highly conserved not only in terrestrial species, from insects to rodents and humans, but also in marine mollusks (Panda et al., 2002; Lowrey and Takahashi, 2000; Shi and Zheng, 2013), which suggests a convergent evolution of circadian timing mechanisms and raises the possibility that the underlying timekeeping mechanism may also be conserved.

Interconnections between circadian and lunar rhythms are found in some marine organisms, such as corals and fishes (Levy et al., 2007; Vize, 2009; Fukushiro et al., 2011; Ikegami et al., 2014). It is suggested that the rhythmic expression of melatonin may serve as a possible transducer between lunar spawning rhythmicity and circadian rhythms in fishes (Ikegami et al., 2014). Consistently, we detected a strong negative relationship in the expression level between melatonin and the clock genes (per and cry), wherein melatonin generally has a 10-fold higher expression level (RPKM) than per and cry. However, the interconnections between circadian rhythms and lunar-related reproductive activity in marine bivalves need to be further examined in physiological and experimental studies. Additionally, the transcriptome survey of clock genes in this study will not only help understand the principles, interactions, and evolution of rhythms in marine species, but will also be useful to measure the effects of climate change or light pollution in the marine ecosystem.

We performed a comprehensive survey to detect phototransduction and circadian genes in the mantle transcriptome of the scallop P. yessoensis, and compared the results with three other bivalves. The results revealed the transcripts of most core members from phototransduction and circadian networks in terrestrial model species, such as arrestin, calmodulin, guanyl cyclase, inaC, opsin, phosducin, recoverin, clock, period, timeless, 
and cycle. The present findings indicate conservation of the timekeeping mechanism from terrestrial species to marine bivalves. Moreover, the identification of clock genes will provide a valuable gene resource for "marine rhythms" and help understand the diversification and evolution of rhythms in marine species.

\section{Conflicts of interest}

The authors declare no conflict of interest.

\section{ACKNOWLEDGMENTS}

Research supported by grants from the Basic Scientific Research Fund of YSFRI (\#2060302201516054), the Zhejiang Provincial Top Key Discipline of Biological Engineering (\#KF2015005), and the Independent Innovation Funds of Shandong Province (\#2013CXC80202).

\section{REFERENCES}

Fukushiro M, Takeuchi T, Takeuchi Y, Hur SP, et al. (2011). Lunar phase-dependent expression of cryptochrome and a photoperiodic mechanism for lunar phase-recognition in a reef fish, goldlined spinefoot. PLoS One 6: e28643. http:// dx.doi.org/10.1371/journal.pone.0028643

Golombek DA and Rosenstein RE (2010). Physiology of circadian entrainment. Physiol. Rev. 90: 1063-1102. http://dx.doi. org/10.1152/physrev.00009.2009

Hardin PE (2005). The circadian timekeeping system of Drosophila. Curr. Biol. 15: R714-R722.http://dx.doi.org/10.1016/j. cub.2005.08.019

Ikegami T, Takeuchi Y, Hur SP and Takemura A (2014). Impacts of moonlight on fish reproduction. Mar. Genomics 14: 59-66.http://dx.doi.org/10.1016/j.margen.2013.11.007

Levitan DR, Fukami H, Jara J, Kline D, et al. (2004). Mechanisms of reproductive isolation among sympatric broadcast-spawning corals of the Montastraea annularis species complex. Evolution 58: 308-323. http://dx.doi. org/10.1111/j.0014-3820.2004.tb01647.x

Levy O, Appelbaum L, Leggat W, Gothlif Y, et al. (2007). Light-responsive cryptochromes from a simple multicellular animal, the coral Acropora millepora. Science 318: 467-470.http://dx.doi.org/10.1126/science.1145432

Li DY, Wu N, Tu JB, Hu YD, et al. (2015a). Expression patterns of melatonin receptors in chicken ovarian follicles affected by monochromatic light. Genet. Mol. Res. 14: 10072-10080. http://dx.doi.org/10.4238/2015.August.21.14

Li J, Grant GR, Hogenesch JB and Hughes ME (2015b). Considerations for RNA-seq analysis of circadian rhythms. Methods Enzymol. 551: 349-367.http://dx.doi.org/10.1016/bs.mie.2014.10.020

Lowrey PL and Takahashi JS (2000). Genetics of the mammalian circadian system: Photic entrainment, circadian pacemaker mechanisms, and posttranslational regulation. Annu. Rev. Genet. 34: 533-562. http://dx.doi.org/10.1146/ annurev.genet.34.1.533

Pairett AN and Serb JM (2013). De novo assembly and characterization of two transcriptomes reveal multiple lightmediated functions in the scallop eye (Bivalvia: Pectinidae). PLoS One 8: e69852-e69852.http://dx.doi.org/10.1371/ journal.pone. 0069852

Panda S, Hogenesch JB and Kay SA (2002). Circadian rhythms from flies to human. Nature 417: 329-335. http://dx.doi. org $/ 10.1038 / 417329 \mathrm{a}$

Parsons GJ, Robinson SMC, Chandler RA, Davidson LA, et al. (1992). Intra-annual and long-term patterns in the reproductive cycle of giant scallops Placopecten magellanicus (Bivalvia: Pectinidae) from Passamaquoddy Bay, New Brunswick, Canada. Mar. Ecol. Prog. Ser. 80: 203-214. http://dx.doi.org/10.3354/meps080203

Schrago CG (2006). An empirical examination of the standard errors of maximum likelihood phylogenetic parameters under the molecular clock via bootstrapping. Genet. Mol. Res. 5: 233-241.

Shi M and Zheng X (2013). Interactions between the circadian clock and metabolism: there are good times and bad times. Acta Biochim. Biophys. Sin. 45: 61-69. http://dx.doi.org/10.1093/abbs/gms110

Genetics and Molecular Research 15 (4): gmr15048726 
Shoguchi E, Tanaka M, Shinzato C, Kawashima T, et al. (2013). A genome-wide survey of photoreceptor and circadian genes in the coral, Acropora digitifera. Gene 515: 426-431.http://dx.doi.org/10.1016/j.gene.2012.12.038

Sugama N, Park JG, Park YJ, Takeuchi Y, et al. (2008). Moonlight affects nocturnal Period2 transcript levels in the pineal gland of the reef fish Siganus guttatus. J. Pineal Res. 45: 133-141. http://dx.doi.org/10.1111/j.1600079X.2008.00566.X

Sun X, Yang A, Wu B, Zhou L, et al. (2015). Characterization of the mantle transcriptome of yesso scallop (Patinopecten yessoensis): identification of genes potentially involved in biomineralization and pigmentation. PLoS One 10: e0122967.http://dx.doi.org/10.1371/journal.pone.0122967

Tessmar-Raible K, Raible F and Arboleda E (2011). Another place, another timer: Marine species and the rhythms of life. BioEssays 33: 165-172. http://dx.doi.org/10.1002/bies.201000096

Vize PD (2009). Transcriptome analysis of the circadian regulatory network in the coral Acropora millepora. Biol. Bull. 216: 131-137.

Vize PD, Embesi JA, Nickell M, Brown DP, et al. (2005). Tight temporal consistency of coral mass spawning at the Flower Garden Banks, Gulf of Mexico, from 1997-2003. Gulf Mex. Sci. 23: 107-114.

Wager-Smith K and Kay SA (2000). Circadian rhythm genetics: from flies to mice to humans. Nat. Genet. 26: 23-27. http:// dx.doi.org/10.1038/79134

Zhang G, Fang X, Guo X, Li L, et al. (2012). The oyster genome reveals stress adaptation and complexity of shell formation. Nature 490: 49-54. http://dx.doi.org/10.1038/nature11413

\section{Supplementary material}

Table S1. Identification of phototransduction and circadian genes in the mantel tissue of Patinopecten yessoensis based on Kyoto Encyclopedia of Genes and Genomes (KEGG) pathway annotations. NMDA, N-methyl D-aspartate; ROR, receptor-related orphan receptors; RXR, Retinoid X Receptor; MAP, mitogen-activated protein.

Genetics and Molecular Research 15 (4): gmr15048726 